\title{
A stable and accurate wavelet-based method for noise reduction from hyperspectral vegetation spectrum
}

\begin{abstract}
Hyperspectral vegetation spectrum is normally contaminated with noise and the presence of noise affects the results of vegetation studies, such as species discrimination and classification, disease detection, stress assessment and the estimation of vegetation's biophysical and biochemical characteristics. Additionally, hyperspectral signals are usually studied using the derivative analysis method that is very sensitive to noise in the data. This study investigates denoising of the hyperspectral vegetation spectrum using different waveletbased methods. A test signal and several real-world vegetation spectra are denoised using four wavelet methods: traditional discrete wavelet transform (DWT); stationary wavelet transform (SWT); lifting wavelet transform (LWT); and a combination of SWT and LWT, which in this paper is called stationary lifting wavelet transform (SLWT). SLWT incorporates the advantages of both SWT and LWT methods, including a translation invariance property and a fast simple algorithm. Experimental results show that SLWT highly outperforms other wavelet-based methods in terms of accuracy and visual quality. Furthermore, this research reveals the following novel results: SLWT 1) for different levels of decomposition of the wavelet transform gives similar results and its denoising results is independent to the selection of decomposition level; 2) generates stable statistical results; 3) can make use of mother wavelets with small filter size (i.e., low-order mother wavelets) that are suitable for preserving subtle features in vegetation spectrum; and 4) its denoising results do not depend on the selection of the mother wavelet when applying low-order mother wavelets.
\end{abstract}

Keywords: Hyperspectral remote sensing; Vegetation spectrum; Noise reduction; Noise removal; Denoising; Stationary wavelet transform; Second generation wavelet 